\title{
Electromagnetic characterization of biological cells
}

François Buret*, Naoufel Haddour, Julie Laforet-Ast, Laurent Nicolas, Ronan Perrussel, Damien Voyer, Noël Burais, Marie Frénéa-Robin, Riccardo Scorretti, Nicolas Siauve

\begin{abstract}
This paper presents the most commonly used method to characterize individual biological cells on a dielectric point of view. It is a force based technique which lays on dielectrophoresis and/or electrorotation. First the principle of these phenomena are described and analyzed with an extension to magnetic forces at the micrometric scale level. Secondly we present an experimental setup which permits to acquire the dielectrophoretic spectrum which is a dielectric signature of a cell. The main dielectric parameters can be deduced by fitting the theoretical response of the cell issued from a dielectric model and the experimental data. At the end we present an improved fitting method which takes advantage of a sensitivity analysis based on a probabilistic approach.
\end{abstract}

Keywords Biological cell, Magnetophoresis, Dielectrophoresis, Electrorotation, Electromagnetic characterization, Parameter extraction.

\section{Caracterização eletromagnética de células biológicas}

Resumo Este trabalho apresenta um dos métodos mais utilizados para caracterização de células biológicas individuais sob o ponto de vista dielétrico. É uma técnica baseada na força produzida por células submetidas a campos magnéticos ou elétricos. Inicialmente, os princípios associados a estes fenômenos são descritos e analisados, incluindo forças magnéticas em escala micrométrica. Posteriormente, é apresentada uma configuração experimental que permite obter um espectro dieletroforético, que é a assinatura dielétrica da célula. Os principais parâmetros dielétricos podem ser deduzidos ajustando-se a resposta teórica obtida do modelo dielétrico e os dados experimentais. Ao final é apresentado um método de ajuste, melhorado por meio da análise de sensibilidade baseada em uma abordagem probabilística.

Palavras-chave Células biológicas, Magnetoforese, Dieletroforese, Eletrorotação, Caracterização eletromagnética, Extração de parâmetros. 


\section{Introduction}

The effects of electromagnetic fields on the human being have become a big concern for the public opinion, which most often suspects bad consequences on health. This dark side is brought to the fore while some positive aspects of these interactions are kept hidden. Besides medical imagery which is commonly used and accepted by people, electromagnetic fields can be an instrument of sophisticated technologies for clinical or biotechnological applications.

This point can be illustrated by the phenomenon called electropermeabilization (often called electroporation). The permeability of the cell membrane to molecules can be transiently increased when an external micro or millisecond electric field pulse is applied on a cell. Under suitable conditions, depending mainly on the pulse parameters, the viability of the cell can be preserved. It is an elegant way to gain access to the cytoplasm and to introduce chosen foreign molecules, without irreversible cell damage. For example, the microbiologists commonly use the electropermeabilization phenomenon in order to perform gene transfers into bacteria by introducing plasmid into the cytoplasm. Now this method is also proposed as an efficient way for drug, oligonucleotides, antibodies and plasmids delivery in vivo. Thus if the microbiologists may use electropermeabilization in a rough way where only the efficiency of the gene transfer is taken into account without any consideration to the rate of lethal effect, it is crucial to monitor and characterize the effect of an applied transient electric field when considering medicinal or veterinary in vivo applications.

Since the permeabilization of cells takes place only where the local field reaches a critical value through the membrane, we are facing a key question: how can we locally control the field, and what happens on both membrane and cellular scales, when an electromagnetic field is applied at the tissue level?

To answer this question, we have to face a large range of sub-questions, some of which being:

- The dielectric characteristic of tissues at the macroscopic level;

- A relevant model of a cell with regard to the problem;

- The dielectric characteristics of the different cell compartments.

The problems non-exhaustively listed above are strongly connected and each of them is a scientific issue for researchers involved in the study of the interaction between electromagnetic fields and living matter. Among these issues, the understanding and the control of this interaction at the cell scale can be seen as the basic requirement to understand also what happens at a higher scale.

This paper is mainly focused on one aspect of the electric field interaction with living cell. It concerns a method that can be implemented to extract the dielectric parameters of a single cell.

\section{Methods}

\section{The Cell}

\section{Basis}

Cells are the elementary units of living creatures. They can be classified into two mains categories:

- Prokaryotic cells which have no nucleus containing the genetic material. Their size is in the range of a few micrometers. Bacteria belong to this category;

- Eukaryotic cells which have a nucleus. They are typically 10 to 100 times larger than prokaryotic cells. Tissues, organs of animals, fungi and plants are built with this kind of cell.

\section{Structure}

Different cell species can present a large variety of shapes and structures but these structures always share two common points: the cytoplasm and the membrane.

The composition of the cytoplasm is slightly different for eukaryotic and prokaryotic cells but it does not matter in the present context. The cytoplasm is a jelly-like substance where water represents up to $70 \%$ of the cell mass. It contains the organelles and the nucleus in the case of an eukaryotic cell (Figure 1);

The membrane is the external envelop of the cell surrounding the cytoplasm. It acts as a selectively permeable barrier, keeping foreign entities out of the cell and its contents inside the cell (the cytoplasm), allowing in the same time selected substances to pass in and out of the cell. It is a structure consisting mainly of phospholipid molecules organized in a double layer, and proteins which control ion exchanges through the membrane. The membrane is thin compared to the size of the cell. Its thickness is in the 2-9 nanometers range (Figure 2). This size contrast is a well known problem in numerical modeling (Meny et al., 2007).

\section{Electromagnetic characteristics}

Most of biological cells are diamagnetic with a volume magnetic susceptibility $\chi$ which corresponds more or less to the value of water near $-10^{-5}\left(\chi=\mu_{\mathrm{r}}-1\right.$ where $\mu_{\mathrm{r}}$ is the relative permeability). If the red blood cells have a higher susceptibility value $\left(-6.5 \cdot 10^{-6}\right)$ due to the small amount of iron that they contain, there are also 


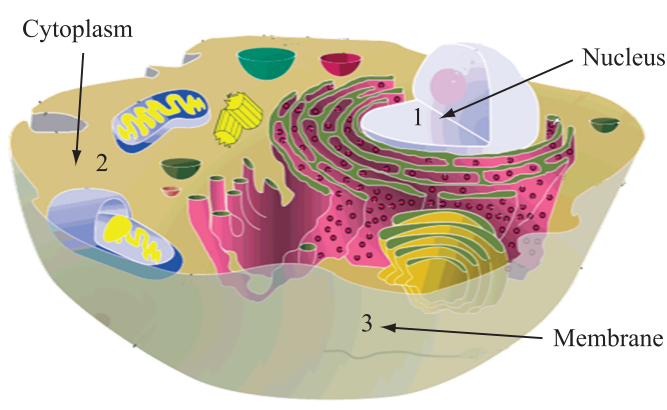

Figure 1. Schematic of a typical animal cell: 1) nucleus; 2) cytoplasm; 3) membrane. From http://en.wikipedia.org/wiki/File:Biological_cell. svg.

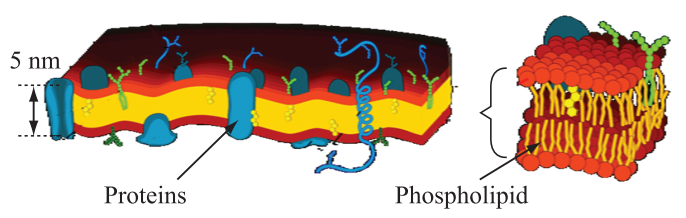

Figure 2. Schematic of the membrane structure. From http://commons. wikimedia.org/wiki/File:Cell_membrane_detailed_diagram_3.svg.

cells which have intrinsic magnetization. For instance Magnetospirillum magneticum is a bacterial species which synthesizes chains of magnetic nanoparticles in the cytoplasm. The level of magnetization is big enough to make the bacteria sensitive to the Earth's magnetic field.

Concerning cell dielectric properties, the description by a constant permeability and a constant conductivity is relevant in the range from $1,000 \mathrm{~Hz}$ to $10^{7} \mathrm{~Hz}$ (Kotnik and Miklavčič, 2000). The two main domains (cytoplasm and membrane) of a cell present a high contrast (Table 1) and play the most significant roles in the global dielectric behavior of the cell. It is the reason why attention is paid to these two regions in terms of modeling in a first approach. Indeed even if the volume of the membrane is negligible compared to the total volume of the cell, this latter has a great influence on the dielectric behavior. The almost high values of relative permittivity $\varepsilon_{\mathrm{r}_{\mathrm{cyt}}}$ and conductivity $\sigma_{\text {cyt }}$ in the cytoplasm are related to its content of water and ions.

\section{Influence of Electromagnetic Fields on a Cell}

Since we want to characterize individual cells, we have to measure the effects due to electromagnetic fields at the cell scale, what implies on the use of micro technologies. The characterization methods presented below are based on the production of forces on a cell when it is submitted to a magnetic or electric field.
Table 1. Dielectric characteristics.

\begin{tabular}{lcccccc}
\hline \multicolumn{1}{c}{ Cell type } & \multicolumn{2}{c}{ Cytoplasm } & & \multicolumn{2}{c}{ Membrane } \\
\cline { 2 - 3 } \cline { 5 - 6 } & $\varepsilon_{\mathbf{r}_{\text {cyt }}}$ & $\begin{array}{c}\sigma_{\text {cyt }} \\
\left(\mathbf{S . m}^{-1}\right)\end{array}$ & & $\varepsilon_{\mathbf{r}_{\text {mem }}}$ & $\begin{array}{c}\sigma_{\text {men }} \\
\left(\mathbf{S . m}^{-1}\right)\end{array}$ \\
\hline Red blood cell & 59 & 0.3 & & 4.4 & $<10^{-6}$ \\
Jurkat cell & 45 & 0.4 & & 6.0 & $3 \cdot 10^{-6}$ \\
\hline
\end{tabular}

\section{Magnetophoresis}

A particle of volume $V_{p}$ and of susceptibility $\chi_{p}$ immersed in a medium of susceptibility $\chi_{m}$ is submitted to a force given by Schenck (2000):

$\vec{F}=\frac{\left(\chi_{p}-\chi_{m}\right)}{2 \mu_{0}} V_{p} \vec{\nabla} B^{2}$

where $B$ is the modulus of the induction field vector and $\mu_{0}$ the permeability of vacuum $\left(4 \pi \cdot 10^{-7} \mathrm{H} / \mathrm{m}\right)$.

The difference between the susceptibilities $\left(\Delta \chi=\chi_{p}-\chi_{m}\right)$ can be positive or negative:

- If $\Delta \chi$ is positive (positive magnetophoresis), the particle is attracted towards the maximum field regions;

- If $\Delta \chi$ is negative (negative magnetophoresis), the particle is attracted towards the minimal field regions.

Equation 1 points out also that (i) the force is independent of the direction of the induction $B$, (ii) the gradient of $B$ is more important than the magnitude of $B$. This last point is fundamental in the context of the cell. Indeed it is usually difficult to expect significant value of a force with diamagnetic material. At the micrometric scale, high values of field gradients can be obtained with a permanent micromagnet array. We can get an order of magnitude of the force on the basis of the following elements $\left(\Delta \chi=10^{-6}\right.$; $B_{\text {max }}=0.25 \mathrm{~T} ; B_{\text {min }}=0 \mathrm{~T}$; typical length $\left.\mathrm{l}=10 \mu \mathrm{m}\right)$ : $\mathrm{F} \approx 10^{4} V_{p}$ (SI units).

By assuming that the main constituent of the particle is water, the value above can be compared to the weight $P$ which is in the same range: $P \approx 10^{4} \mathrm{~V}_{\mathrm{p}}$ (SI units).

The susceptibility contrast can be also increased by adding paramagnetic ions (Gd-DTPA: Gadoliniumdiethylenetriaminepenta-acetic acid is a paramagnetic complex used as a MRI contrast agent). The susceptibility of the medium can be measured thanks to a magnetic balance and thus we have a method to evaluate indirectly the susceptibility of a particle (cell) by measuring the velocity of the particle.

Nevertheless it must be noticed that the micromagnet array presented in Figure 3 is not well suited for the measurement of the susceptibility and is more likely to be used in cell positioning applications. 


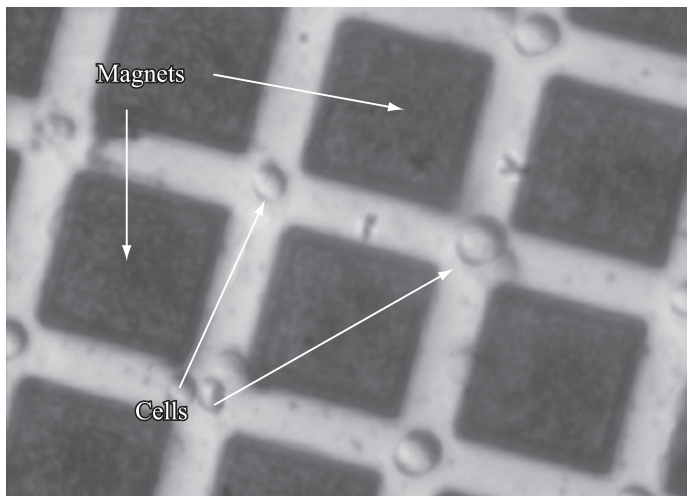

Figure 3. Yeast cells submitted to negative magnetophoresis. Matrix of square magnets (CoPt plots, thickness: $8 \mu \mathrm{m}$, side length: $25 \mu \mathrm{m}$, separated by $10 \mu \mathrm{m}$ gaps). Suspension medium: Aqueous solution containing 20 mM Gd-DTPA (Frenea-Robin et al., 2008).

The technique of cell and particle tracking velocimetry for cell magnetization measurement was carefully described by Moore et al. (2004) and Häefeli et al. (2002). The use of permanent magnets instead of coils and currents takes advantage of the fact that there is no heating of the medium and the cell. Nevertheless, in this case the only free parameter is the susceptibility $\left(\chi_{\mathrm{m}}\right)$ of the medium.

\section{Dielectrophoresis (DEP)}

We consider a spherical particle (radius $R$, permittivity $\varepsilon_{p}$ and conductivity $\sigma_{p}$ ) immerged in a medium (permittivity $\varepsilon_{m}$ and conductivity $\sigma_{m}$ ) and exposed to a non-uniform electric field $E$. It is submitted to a force given by the expression (Hughes, 2002):

$$
\vec{F}=2 \pi \varepsilon_{m} R^{3} \operatorname{Re}[K(\omega)] \vec{\nabla} E_{r m s}^{2}
$$

where $K$ is the Clausius-Mossotti factor which depends on the complex permittivities and is expressed as:

$$
K(\omega)=\frac{\varepsilon_{p}^{*}-\varepsilon_{m}^{*}}{\varepsilon_{p}^{*}+2 \varepsilon_{m}^{*}}
$$

with

$$
\varepsilon^{*}(\omega)=\varepsilon-j \frac{\sigma}{\omega}
$$

where $\omega$ is the pulsation of the electric field.

Equation (1) is similar to (2) and the main remarks which have been pointed out for magnetophoresis are still pertinent:

- Insensitivity of the force to the direction of the electric field;

- The field gradient is an important parameter.

We can also define (Figure 4):
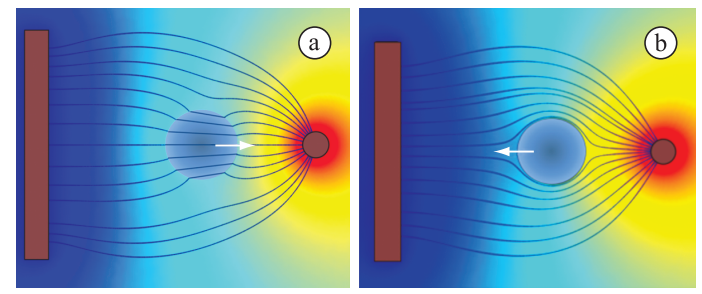

Figure 4. a) Positive, and b) negative dielectrophoresis. The arrows indicate the direction of the force.

- Positive dielectrophoresis when $\operatorname{Re}[K(\omega)]$ is positive. The particle is attracted toward regions with high field values. In this case the global polarization of the particle has the same direction as the electric field;

- Negative dielectrophoresis when $\operatorname{Re}[K(\omega)]$ is negative. The force is directed towards low field region and the direction of the particle polarization is opposite to the field.

Practically, high gradient value of $E$ can be produced by rectangular interdigitated electrodes (Figure 5). It must be noticed that electric field values up to $10^{5} \mathrm{~V} / \mathrm{m}$ can be obtained easily with an applied voltage of 10 volts and a gap in the range of $100 \mu \mathrm{m}$. $\mathrm{AC}$ operation, which is possible due to the dependence of the force on the square of the field modulus, avoid electrolysis of the medium and electrophoretic effects.

\section{Electrorotation (ROT)}

Besides dielectrophoresis described above which is more precisely named conventional dielectrophoresis (c-DEP), another polarization effect, called electrorotation can be obtained in a uniform rotating electric field.

When a rotating field with a constant modulus is applied to a particle, this latter is submitted to a torque which is given by the following expression (Jones, 2003):

$\vec{\Gamma}=-4 \pi \varepsilon_{m} R^{3} \operatorname{Im}[K(\omega)] E^{2}$

where $\omega$ is the rotation speed of the field.

The expression of the torque shows that electrorotation only exists if at least one of the two materials (medium or cell) presents energy losses. The sign of the torque can be positive (co-ROT) or negative (anti-ROT) refered to the rotation of the electric field. The rotation field can be produced by a system of four electrodes fed with an equilibrated quadri-phase voltage. The field map on Figure 6 shows that the modulus is almost constant around the center of the electrode system (white circle). 


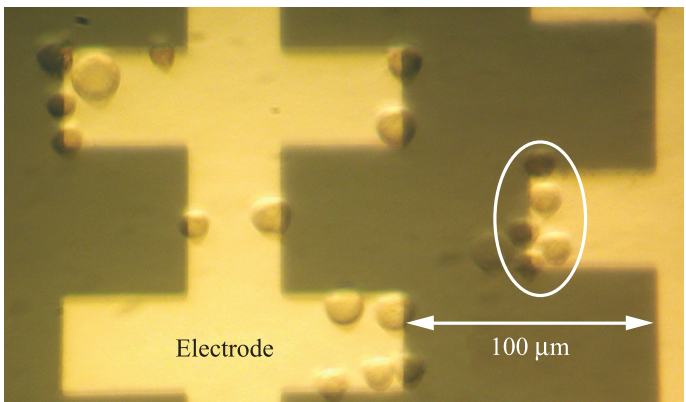

Figure 5. Modulus of the electric field for one motif of the electrodes (up) and positive dielectrophoresis for Jurkat cells (down). High field region is circled.

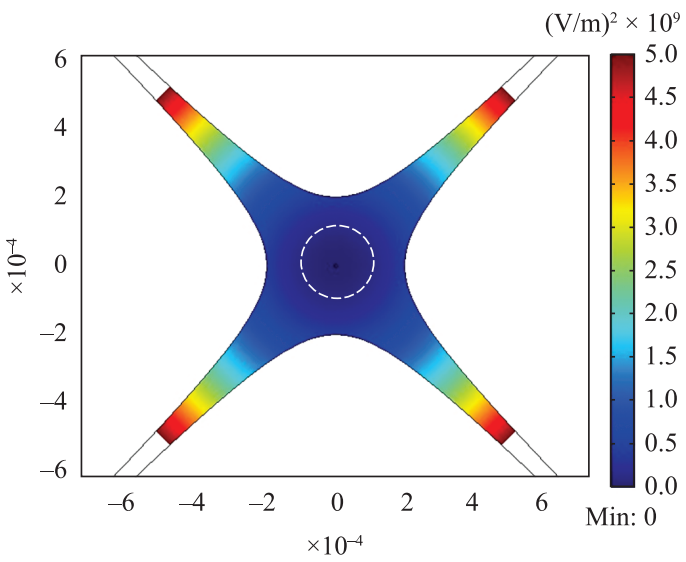

Figure 6. Map of the square of the field modulus for polynomial electrodes.

This system also permits to perform c-DEP in the region between two electrodes by simply changing the feeding mode of the electrodes: for example, top and down electrodes to $+\mathrm{V}$ and left and right electrodes to $-\mathrm{V}$ (Figure 7). In Figure 7 we see latex particles $(R=12 \mu \mathrm{m})$ submitted to negative dielectrophoresis and which are grouped in the low field domain in the center of the electrodes while yeast cells $(R=3 \mu \mathrm{m})$ are attracted towards the high field regions.

\section{Characterization Method Using c-DEP/ROT}

\section{Principle}

As we stated before, a biological cell can be reasonably described by the dielectric properties of the two main domains (cytoplasm and membrane). From Equation 4 it can be deduced that each of these domains has a transition frequency $f_{t}$ defined by:

$f_{t}=\frac{\sigma}{2 \pi \varepsilon}$

For frequencies above $f_{t}$ these materials can be considered as an insulating medium while below $f_{t}$

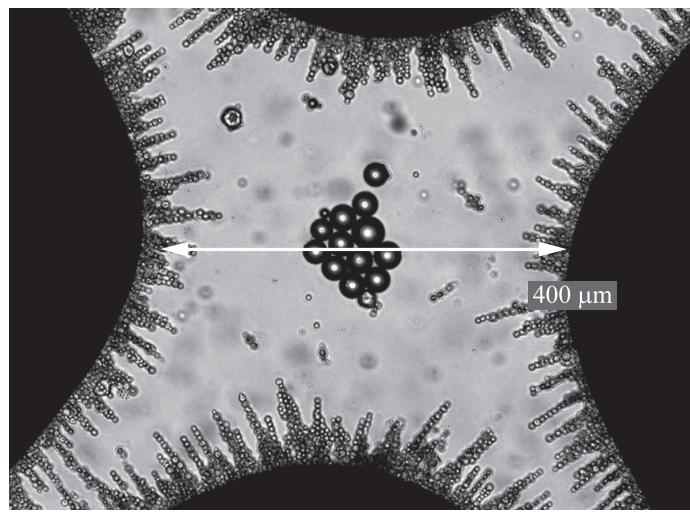

Figure 7. Experiment of c-DEP in polynomial electrodes.

the medium is mainly conductive. Considering the values given in Table 1, the transition frequency of the membrane is much lower than the one of the cytoplasm. This fact has two consequences. On a fundamental point of view, the Maxwell-Wagner interfacial polarization is important for it allows getting high value of the dipolar moment and then facilitates the creation of efficient forces. On a practical point of view, the behavior of the global cell varies with the frequency of the applied field.

To link the dielectric property of a biological cell to the expression of the force and the torque established for a homogeneous particle, we need to use a model. The simplified model of a biological cell presented on Figure 8 (left) is equivalent to a homogeneous sphere with a complex permittivity $\varepsilon_{p}^{*}$ (Irimajiri et al., 1979).

$\varepsilon_{p}^{*}=\frac{R \varepsilon_{m e n}^{*} \varepsilon_{c y t}^{*}}{R \varepsilon_{m e n}^{*}+e \varepsilon_{c y t}^{*}}$

At low frequency the dielectric property are governed by the membrane $\left(\varepsilon_{p}^{*} \approx \frac{R}{e} \varepsilon_{m e n}^{*}\right)$ while at high frequency the cytoplasm is dominant $\left(\varepsilon_{p}^{*} \approx \varepsilon_{c y t}^{*}\right)$. It must be noticed that with the usual range of the ratio $R / e$ (around 1,000), the apparent relative permittivity can be very high (several thousands) which renders a high polarization level of the cell as mentioned above. The theoretical dielectrophoretic spectrum which corresponds to the variation of the Clausius-Mosotti factor $K(\omega)$ versus the frequency can be evaluated. The spectrum drawn on Figure 9 shows that different behaviors can be obtained by varying the frequency. The ROT spectrum exhibits both anti-field and cofield resonances. At low frequency we have negative c-DEP and anti-ROT then above $200 \mathrm{kHz}$ positive c-DEP and finally above $4 \mathrm{MHz}$ co-ROT. The shape of this spectrum depends obviously on the dielectric parameter $\sigma_{c y t}, \varepsilon_{c y t}, \sigma_{m e n}, \varepsilon_{m e n}$ and the geometrical 
parameters $e, R$. Inversely these parameters can be deduced from the experimental acquisition of the dielectophoretic spectrum. It must not be forgotten that the spectrum is also dependent of the dielectric characteristics of the medium. These characteristics are an adjustment parameter in the range where the media stay compatible with cell viability. This spectrum can be obtained through cell speed measurement in c-DEP and/or ROT experiments. Assuming that the viscosity of the medium is $\eta$ :

$$
\begin{aligned}
& \operatorname{Re}(K(\omega))=\frac{3 \eta \mathrm{v}(\omega)}{\varepsilon_{m} R^{2}\left|\nabla E_{r m s}^{2}\right|} \\
& \operatorname{Im}(K(\omega))=-\frac{2 \eta \Omega(\omega)}{\varepsilon_{m} E^{2}}
\end{aligned}
$$

where $\mathrm{v}(\omega)$ and $\Omega(\omega)$ are respectively the measured velocity and the measured rotation speed.

\section{Results}

\section{Experimental setup}

We have developed a platform to acquire the dielectrophoretic spectrum. The microelectrode structure used in the c-DEP or ROT experiments is composed of four polynomial electrodes disposed in a circular arrangement (Figure 10). Those electrodes are powered by 4 generators which can deliver sine-wave voltages from a few $\mathrm{Hz}$ to $80 \mathrm{MHz}$ with appropriate phases. Visualization of the applied voltage is achieved thanks to a wide band oscilloscope whose input impedance can be set to $50 \mathrm{ohms}$ for impedance matching. Cell motion is observed under an inverted microscope and image acquisition is performed by a high speed camera. These different functions are PC-controlled via GPIB interfaces (Figure 11). This permits in particular to maintain the applied voltage constant over the whole frequency range, despite the variations of the micro device impedance. The acquisition of a dielectrophoretic spectrum by measuring the cell speed with a stopwatch is a tedious work. We have developed a software that analyzes the video performed with the high speed camera to automatically extract the cell rotation rate. Figure 12 presents the variation of the rotation velocity for viable and non viable cells.

\section{Parameters extraction}

Attention is more focused on the ROT spectrum, which shape is complex. Thus this spectrum can give more information on the dielectric parameters than the c-DEP spectrum. On a practical point of view, the rotation velocity is measured in a frequency range wide enough to take into account the interesting zones of the ROT spectrum: the two resonances and the transition frequency.

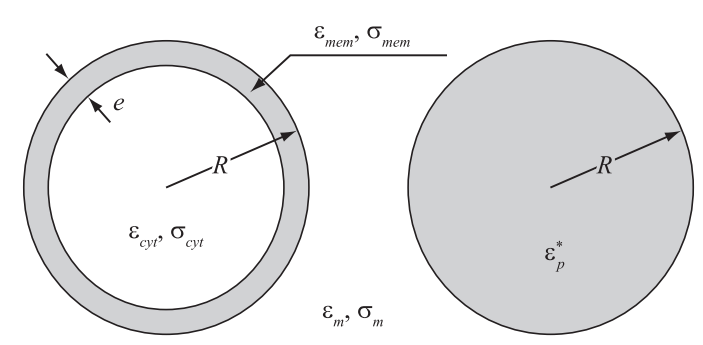

Figure 8. Dielectric model of a single cell.

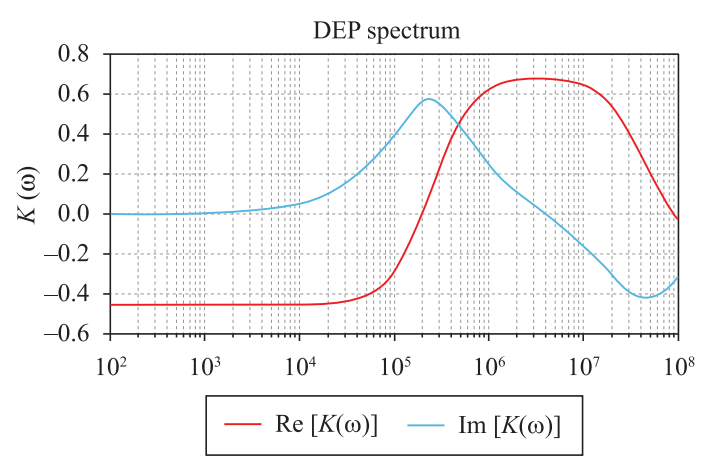

Figure 9. Theoretical dielectrophoresis spectrum of a Jurkat cell, real and imaginary part. See Table 1 for the dielectric properties of the cell; $\sigma_{m}=50 \mathrm{mS} / \mathrm{m}$ and $\varepsilon_{m}=80 \varepsilon_{0}$ for the medium. $R=5 \mu \mathrm{m}, e=5 \mathrm{~nm}$.

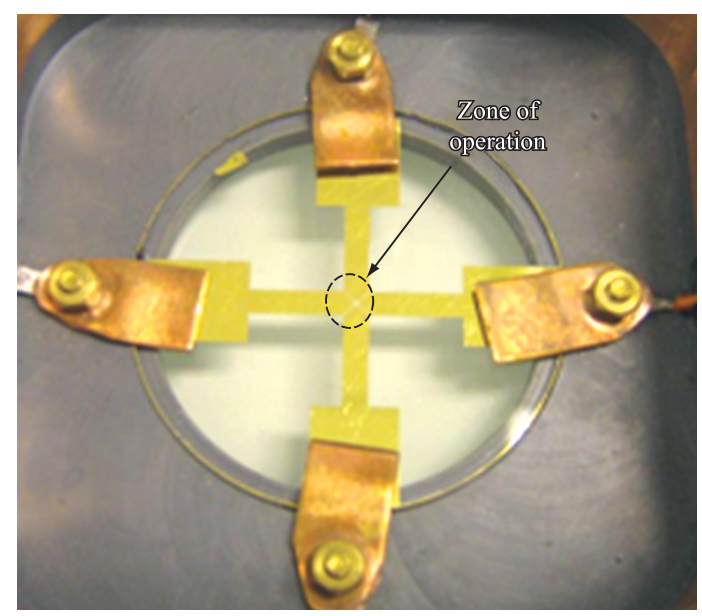

Figure 10. Photo of the microsystem.

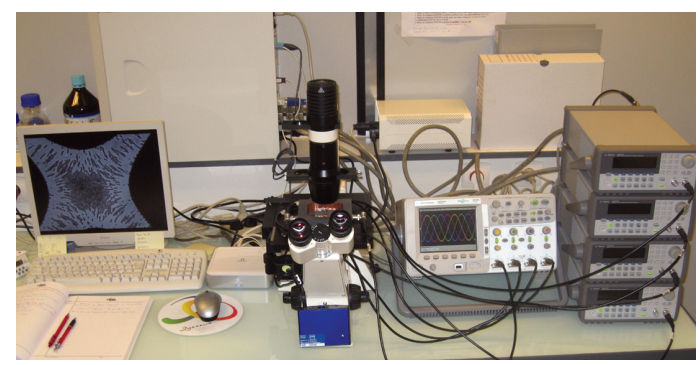

Figure 11. Platform for dielectrophoretic spectrum acquisition. 
We are facing a problem with 8 parameters: $\sigma_{c y t}$, $\varepsilon_{c y t}, \sigma_{m e n}, \varepsilon_{m e n}, e, R, E$ and $\eta$. The typical dimension $R$ of the cell can be acquired by direct visualization (microscopy). The electric field $E$ can be obtained by simulation and $\eta$ can be measured using a viscosimeter. Generally people work directly on the $\Omega(\omega)$ curve, the so-called ROT spectrum (sometimes assimilated to the dielectrophoretic spectrum by misuse of language).

$\Omega(\omega)=-\beta_{R O T} \varepsilon_{m} \operatorname{Im}(K(\omega))$

where $\beta_{R O T}$ integrates the dependence for $E$ and $\eta$. This term has no direct interest for the dielectric model but has a direct influence on the measured values $\Omega_{\text {exp }}(\omega)$. Equation 7 shows also that we can not determine $\sigma_{m e n}, \varepsilon_{m e n}$ and $e$ separately but only the ratio $\sigma_{\text {men }} / e$ and $\varepsilon_{m e n} / e$. Finally the mathematic problem consists in extracting 5 parameters: $\sigma_{c y}, \varepsilon_{c y t}, \sigma_{m e n} / e, \varepsilon_{m e n} / e$, and $\beta_{\text {ROT }}$. The basic method to achieve this goal has been proposed by Gascoyne et al. (1995). The experimental ROT spectrum $\Omega_{\text {exp }}$ is fitted with a simulated one $\Omega_{\text {sim }}$ calculated thanks to Equation 10. This is done by minimizing the distance between experimental and simulated data at the different frequency points $f_{i}$ (pulsation $\omega_{i}=2 \pi f_{i}$ ). This discrete least-square minimization problem can be summarized as follows:

$\min \left\{\sum_{i}\left|\Omega_{\exp }\left(\omega_{i}\right)-\Omega_{\text {sim }}\left(\omega_{i}\right)\right|^{2}\right\}$

the different parameters being included in their respective prospective range of values.

In a previous study conducted by Gascoyne et al. (1995) the minimization procedure was achieved by using the Nelder-Mead simplex optimization method. Nevertheless tests have given evidence that the reliability of this method is somewhat weak. We have proposed an improvement of this method by (Voyer et al., 2009):

- Weighting the summation terms in Equation 11 by an appropriate frequency-dependent coefficient $\alpha_{i}$.

$\min \left\{\sum_{i} \alpha_{i}\left|\Omega_{\exp }\left(\omega_{i}\right)-\Omega_{\text {sim }}\left(\omega_{i}\right)\right|^{2}\right\}$

- Applying gradually the minimization process to the different parameters, using for each of them the suitable weighted spectrum instead of extracting all the parameter in one single step.

The underlying idea of this improved approach is that each parameter has an influence on the spectrum which varies with frequency as mentioned before. To evaluate this influence we have performed a sensitivity analysis (variance based) in order to ascertain how much the ROT spectrum depends on the different parameters. Figure 13 and Figure 14 illustrate this point. Figure 13 represents the dispersion of the

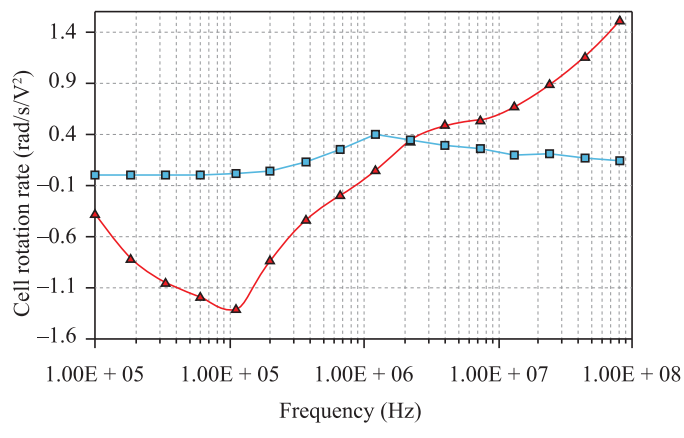

$\triangle$ Viable yeast cells $\rightarrow-$ Non-viable yeast cells

Figure 12. Experimental ROT spectrum for yeast cell (Saccharomyces cerevisiae). Medium: $\varepsilon_{r m}=80, \sigma_{m}=1.1 \mathrm{mS} / \mathrm{m}$.

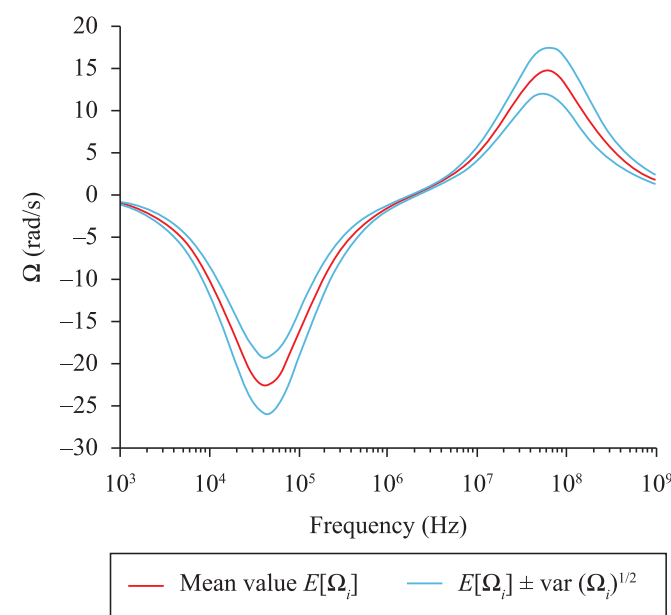

Figure 13. Mean value of the spectrum and its dispersion (standard deviation). $\sigma_{c y t} \in[0.6,0.9] \mathrm{S} / \mathrm{m}, \varepsilon_{r c y t} \in[60,140], \sigma_{m e m} / e \in[800$, $1,000] \mathrm{S} / \mathrm{m}^{2}, \varepsilon_{\text {mem }} \in[20,30] \mathrm{F} / \mathrm{m}^{2}, \beta_{R O T} \in[30,50] \mathrm{rad} / \mathrm{s}, R=15 \mu \mathrm{m}$, $\varepsilon_{r m}=80, \sigma_{m}=50 \mathrm{mS} / \mathrm{m}$.

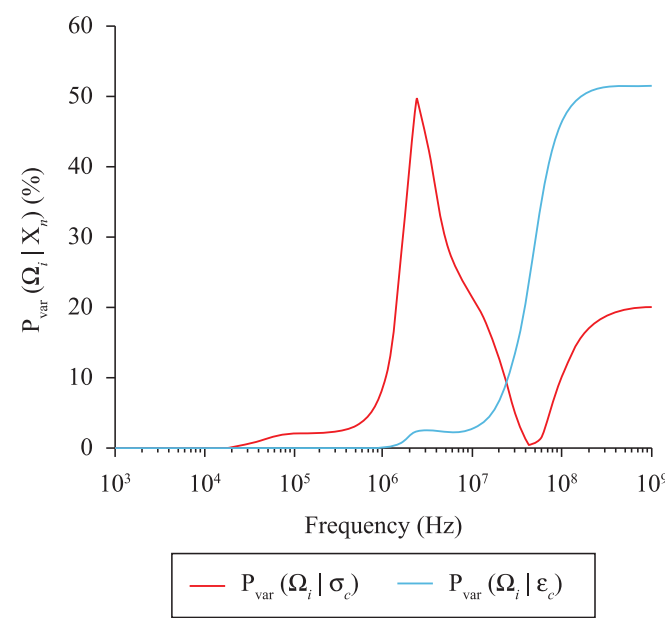

Figure 14. Relative partial variance $\mathrm{P}_{\mathrm{var}}\left(\Omega_{i} \mid \sigma_{c}\right)$ and $\mathrm{P}_{\mathrm{var}}\left(\Omega_{i} \mid \varepsilon_{r c}\right)$ due to $\sigma_{c y t}$ and $\varepsilon_{r c y t}$ (partial variance divided by the variance at the considered frequency). 
spectrum $\left(\Omega_{i}=\Omega\left(\omega_{i}\right)\right)$ when the parameters vary in their physical intervals. We observe that the dispersion is more important around the peaks but also that the peak frequency remains nearly constant. Figure 14 shows how much $\sigma_{c y t}$ and $\varepsilon_{c y t}$ impact the ROT spectrum. As expected, the dielectric properties of the cytoplasm have an impact mainly in the high frequency range.

When the extraction of one of the parameters is under consideration, the relative partial variance of this parameter is a good candidate to be the weight term $\alpha_{i}$ in Equation 12. The parameters are extracted in 4 successive steps with regard to the partial variances where they are involved. In order to evaluate the efficiency of this approach, a numerical experiment has been carried out. From Equation 10200 samples of spectra have been built choosing randomly the parameters in the bounded interval (Figure 13). The parameters are then extracted using the weighted and the unweighted minimization. It appears that the weighted minimization gives more accurate results. For example the value of $\varepsilon_{c y t}$ has been recovered with a confidence limit around $8 \%$ (weighted) and 15\% (unweighted).

\section{Conclusion}

The characterization of biological cells is still a domain in development requiring the implementation of sophisticated experimental techniques associated to judicious numerical methods than can be still widely improved.

As stated in the introduction a better knowledge of the behavior of a cell is one way (but not the only one) to understand what happens at the tissue level. But now we have the possibility to go deeper in the dimension scale. Indeed simulations in molecular dynamics permit to study what happens at the membrane level when this latter is submitted to an electric constraint. Among all the information we can get from these simulations, the permittivity and the conductivity of the membrane can be extracted.

\section{References}

Frenea-Robin M, Chetouani H, Haddour N, Rostaing H, Laforet J, Reyne G. Contactless diamagnetic trapping of living cells onto a micromagnet array. In: EMBS 2008: Proceedings of the IEEE Engineering in Medicine and Biology Society; 2008; Vancouver. Vancouver; 2008. p. 3360-3.

Gascoyne PRC, Becker FF, Wang X-B. Numerical analysis of the influence of experimental conditions on the accuracy of dielectric parameters derived from electrorotation measurements. Bioelectrochemistry and Bioenergetics. 1995; 36:115-25. http://dx.doi.org/10.1016/0302-4598(94)05015-M

Häfeli UO, Ciocan R, Dailey JP. Characterization of magnetic particles and microspheres and their magnetophoretic mobility using a digital microscopy method. European Cells and Materials. 2002; 3(supp 2):24-7.

Hughes MP. Strategies for dielectrophoretic separation in laboratory-on-a-chip systems. Electrophoresis. 2002; 23(16):2569-82. http://dx.doi.org/10.1002/15222683(200208)23:16\%3C2569::AID-ELPS2569\%3E3.0.CO;2-M

Irimajiri A, Hanai T, Inouye, A. A dielectric theory of "multistratified shell" model with its application to a lymphoma cell. Journal of Theoretical Biology. 1979; 78(2):251-69. http://dx.doi.org/10.1016/0022-5193(79)90268-6

Jones TB. Basic theory of dielectrophoresis and electrorotation. IEEE Engineering in Medicine and Biology Magazine. 2003; 22(6):33-42. PMid:15007989. http://dx.doi. org/10.1109/MEMB.2003.1304999

Kotnik T, Miklavčič D. Theoretical evaluation of the distributed power dissipation in biological cells exposed to electric fields. Bio Electro Magnetics. 2000; 21(5):385-94. http://dx.doi.org/10.1002/1521186X(200007)21:5\%3C385::AID-BEM7\%3E3.0.CO;2-F

Meny I, Burais N, Buret F, Nicolas L. Finite-element modeling of cell exposed to harmonic and transient electric fields. IEEE Transactions on Magnetics. 2007; 43(4):1773-6. http://dx.doi.org/10.1109/TMAG.2007.892517

Moore LR, Milliron S, Williams PS, Chalmers JJ, Margel S, Zborowski M. Control of magnetophoretic mobility by susceptibility-modified solutions as evaluated by cell tracking velocimetry and continuous magnetic sorting. Analytical Chemistry. 2004; 76:3899-907. PMid:15253623. http://dx.doi.org/10.1021/ac049910f

Schenck JF. Safety of strong, static magnetic fields. Journal of Magnetic Resonance Imaging. 2000; 12(1):2-19. http:// dx.doi.org/10.1002/1522-2586(200007)12:1\%3C2::AIDJMRI2\%3E3.0.CO;2-V

Voyer D, Frénéa-Robin M, Buret F, Nicolas L. Improvements in the extraction of cell electric properties from their electrorotation spectrum. Bioelectrochemistry. 2010; 79(1):25-30. PMid:19892606. http://dx.doi.org/10.1016/j. bioelechem.2009.10.002

\section{Authors \\ François Buret, Naoufel Haddour, Julie Laforet-Ast, Laurent Nicolas, Ronan Perrussel, Damien Voyer \\ Laboratoire Ampère CNRS UMR 5005, Université de Lyon, Ecole Centrale de Lyon, 36 Avenue Guy de Collongue, 69134, ECULLY Cedex, France}

Noël Burais, Marie Frénéa-Robin, Riccardo Scorretti, Nicolas Siauve

Laboratoire Ampère CNRS UMR 5005, Université de Lyon, Universite Lyon I, 36 Avenue Guy de Collongue,

69134, ECULLY Cedex, France 